\title{
A rigorous approach to analyze bulk and coreflood foam screening tests
}

\author{
Negar Hadian Nasr ${ }^{1}$ (D) . Syed M. Mahmood ${ }^{1} \cdot$ Hamed Hematpur $^{2}$
}

Received: 20 February 2018 / Accepted: 31 August 2018 / Published online: 6 September 2018

(c) The Author(s) 2018

\begin{abstract}
There are many uses of foam in petroleum industry yet there is no dependable industry standard on screening a wide variety of foaming surfactants available for a particular application. This study aims to fill this gap. Three anionic foaming surfactants were characterized and tested with the two commonly used screening methods at room temperature and oil-free conditions. The results were comprehensively analyzed to compare their foaming performance. The analysis is more comprehensive than previously reported and covers many foaming attributes (peak and residual foamability, foam longevity, and rate of decay). The three surfactants for possible foaming applications in sandstone reservoirs were selected, and their foamability and foam stability performances were experimentally determined by bulk foam stability tests and coreflood tests. All methods agreed on the ratings of the three surfactants for peak and residual foaming attributes as follows in the following order of effectiveness: MFOMAX, AOS, and ENORDET. However, they broadly disagreed on ratings for other characteristics including onset of foaming, the time required for peak foaming, foam longevity, and foam decay rate. In conclusion, the screening tests revealed that the simple and faster bulk foam stability test could be cautiously used to screen out the poor performers to narrow the range of acceptable surfactants. Also, the new and rigorous analysis technique presented in this paper offers more insight than conventional half-life test.
\end{abstract}

\section{Graphical abstract}

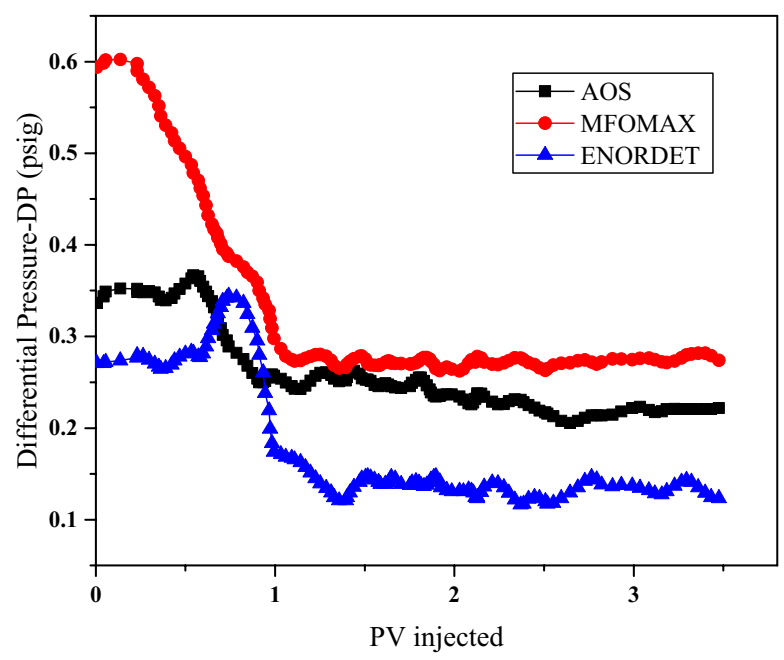

Extended author information available on the last page of the article 

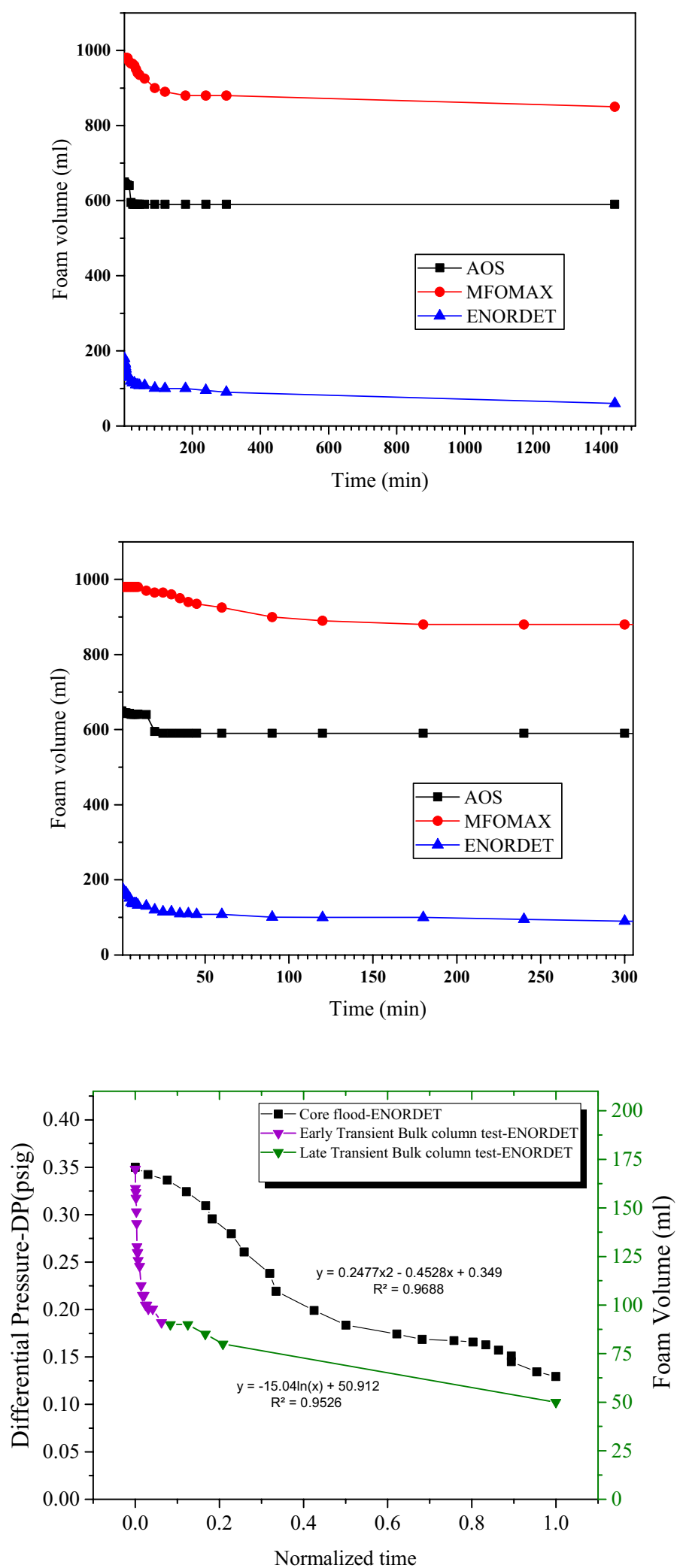


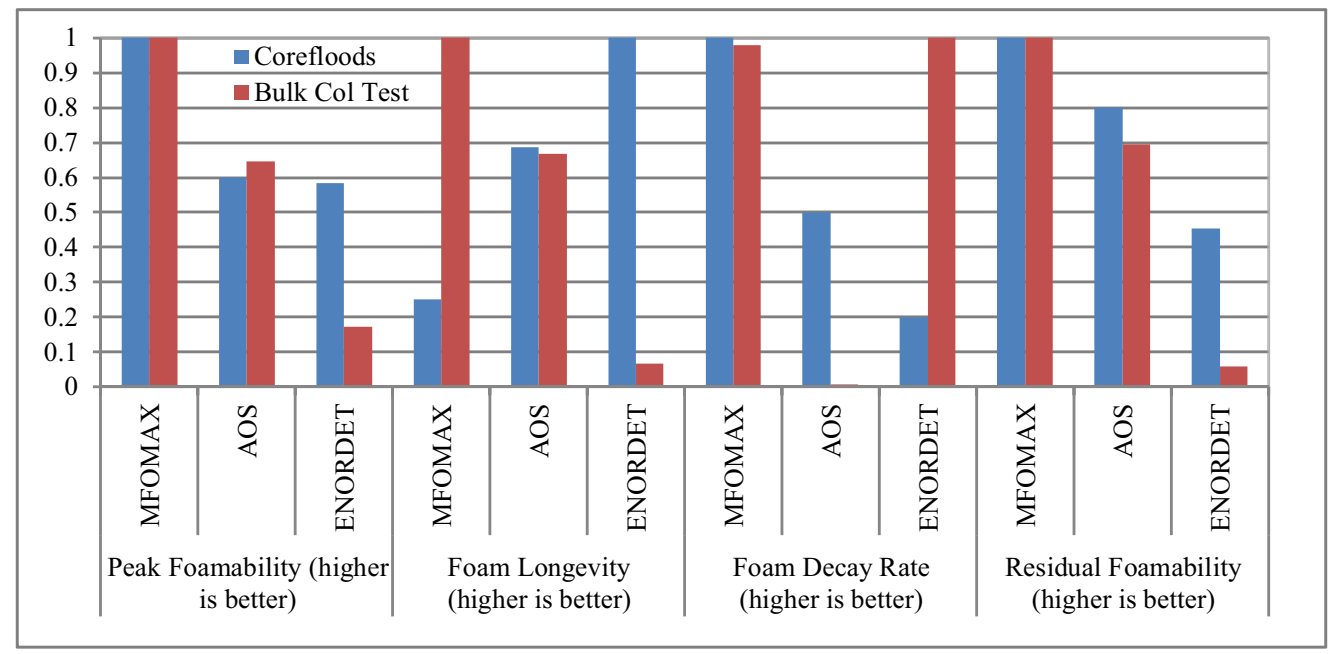

Keywords Bulk foam stability test $\cdot$ Core flood $\cdot$ Peak and residual foamability $\cdot$ Foam longevity $\cdot$ Rate of foam decay

\section{Introduction}

The gas flooding is one of the most accepted and widely used methods for enhancing oil recovery (EOR) from oil reservoirs (Green and Willhite 1998; Lake 1996; Franklin and Orr 2007). However, since the viscosity of the gas is an order of magnitude lower than liquid, gas often fails to displace oil in a piston-like manner and tends to by-pass oil in the form of fingers and channels, especially when high permeability streaks present. Another problem encountered by gas injection is gravity segregation due to the low density of gas vs. oil, which causes gas to by-pass oil through gravity tongues (Namani et al. 2012). To overcome these problems, operators often inject a small slug of foaming surfactant before beginning gas injection with the aim of generating foam to reduce the mobility of injected gas as many studies have suggested (Bond et al. 1958), (Hirasaki 1985). This EOR method is called single-cycle surfactant-alternating-gas (SAG) or unsteady state test.

A large number of surfactants are available commercially for field applications of SAG. Also, major oil companies have developed their proprietary surfactants tailored to the specific reservoir conditions. Choosing a single surfactant that will perform best for a given reservoir is, however, not an easy and deterministic task. The process of selecting a suitable surfactant for the candidate reservoir out of a large number is non-deterministic. The coreflood tests, when conducted under reservoir conditions, are considered to be the most authentic method due to the closely matching conditions under which foam is generated and propagated in the reservoir. In these tests, a small plug of the cylindrical core (typically 3-inch long and of 1.5 inches in diameter) was saturated with reservoir fluids, after which a small slug of surfactant injected, followed by the gas injection.

Unfortunately, coreflood tests are expensive and timeconsuming, hence not suitable for screening from a large number of surfactants (Jones et al. 2016). The standard industry practice is to perform the initial screening of surfactants in glassware commonly available in the lab by injecting gas into a surfactant solution to generate foam, or using a blender, in the absence of sand grains. These tests are called bulk foam stability tests because foam generates and decays outside porous media. These tests are simpler, faster, and cheaper. Some automated systems are commercially available (TECLIS FoamScan 2018) that use image analysis to determine the foam height vs. time (Li et al. 2014). Final selection of a surfactant is made by performing coreflood tests on a few outstanding performers from the bulk foam stability tests.

There is no general agreement or correlation between the foam stability in bulk foam stability tests and the coreflood tests. Several studies have suggested that the bulk foam stability tests are not as reliable as coreflood tests in accurately screening surfactants for field applications (Andrianov et al. 2012; Mannhardt et al. 2000; Farajzadeh et al. 2010; Bergeron et al. 1993). Previous studies hypothesized the reasons for the discrepancies based on the physical differences. The primary reason alluded was that foam morphology inside porous media is different than "free" foam outside porous media due to the confined and heterogeneous pore spaces (Hanssena and Kristiansen 1994). The foam in porous media encounters critical capillary pressure and critical water saturation which are highly foamicidal. In bulk foam test, on the other hand,

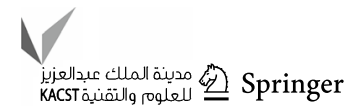


the gravity drainage of surfactant solution plays a dominant role as it causes the lamellae surrounding foam bubbles gets thinner, thereby the results are increasing the rate of gas diffusion from smaller bubbles (which are at higher pressure due to higher capillary pressure) to the larger ones. This diffusion causes the coarsening of bubble sizes with time as the smaller bubbles gradually coalesce into larger bubbles. Finally, the foam becomes dry and takes polyhedral structure (Jones et al. 2016). Recently, the coarsening behavior has been investigated in porous media by Jones (2018) at both pores and core scales using micromodel and sandstone core. They noted the change in bubble count over time and observed that the confining pore walls have a significant effect on foam's coarsening behavior; the coarsening was quicker (coarsening stopped earlier) in smaller pores and pore-throat environments such as the core as compared to the micromodel (Jones 2018).

Another significant difference of bulk foam stability tests is that it is performed under static conditions, whereas coreflood tests are performed at flowing conditions. Foam stability during coreflooding is thus a function of many parameters such as interstitial fluid flow velocities, the method of gas and surfactant injection, and the gas to liquid injection ratio $\left(f_{\mathrm{g}}\right.$, the foam quality) (Zhang et al. 2009).

Some studies showed a positive correlation between bulk foam stability tests and coreflood stability tests under oilfree conditions. Vikingstad and Aarra (2009) used a blender to disperse air for generating foam at a somewhat higher temperature $\left(50^{\circ} \mathrm{C}\right)$. They noted the decrease in foam height with time and compared results with coreflood stability tests in which $\mathrm{N}_{2}$ and surfactant solution were simultaneously injected at a moderate rate and pressure to yield a transitional foam quality $\left(q_{\mathrm{T}}=40 \mathrm{ml} / \mathrm{h}, P_{\mathrm{inj}}=120 \mathrm{bar} / 1764 \mathrm{psig}\right.$, and $F_{\mathrm{g}}=80 \%$ ). They observed that the foam stability and strength were similar in both bulk foam stability tests and coreflood stability tests in oil-free conditions. Also, Jones et al. (2015) suggested using bulk foam stability tests as a proxy to coreflood stability tests for screening foaming surfactants.

Some studies showed a positive correlation under oil-free conditions but failed to show a consistent correlation in the presence of oil (Mannhardt et al. 2000; Jones et al. 2015; Vikingstad and Aarra 2009). Whereas Van der Bent (2014) concluded that bulk foam stability tests could be used to infer surfactant performance in coreflood in the absence of oil, however, he could not find a good correlation between the two methods in the presence of oil (Bent and Der 2014). Osei-Bonsu et al. (2017) conducted a series of foam stability experiments using both micromodel and porous media and found that stability of foam in the presence of oil at bulk scale does not necessarily define its effectiveness in porous media (Osei-bonsu et al. 2017). The ambiguity of bulk foam stability tests in the presence of oil as observed by many investigators precludes them to be used as the sole method for screening surfactants for field applications.

The published studies have demonstrated the superiority of coreflood stability tests over the bulk foam stability tests. However, it is not possible to discard the later altogether because of practical reasons. It is not feasible to screen a large number of surfactants using coreflood which require expensive specialized test equipment and take a few days to conduct a single test vs. bulk foam stability tests that use glassware commonly available in laboratories and take only a few hours for a test. Because of the bulk foam stability test's simplicity and low cost, several screening systems can also run in parallel. Also, some studies show a positive correlation between the two. More studies are needed in an attempt to understand how the bulk foam stability tests can be utilized more intuitively, albeit as an initial screening tool. With this aim in mind, the current study looks into different and more in-depth ways of comparing the two methods of screening. It should be re-emphasized here that this study focuses on oil-free conditions only since studies in the past showed a positive correlation between bulk and core tests in the absence of oil while the use of bulk foam stability test for screening surfactants is ambiguous in the presence of oil.

This study shows the need to examine new attributes of foaming while analyzing both bulk foam tests and core flood results since an understanding of all possible attributes is essential for correctly selecting a suitable candidate surfactant. The "half-life" concept of the bulk test does not directly correlate with corefloods and discrepancies between individual attributes were found while analyzing the results of the two tests. Also, the delay in onset of foaming and time required to reach peak foaming were two of the attributes that could be determined in coreflood tests but not in bulk foam stability tests.

\section{Materials and methodology}

A brief description of the materials used in this study is provided here along with an elaborate discussion on the methodology of the tests.

\section{Brine preparation}

The salinity of the synthetic brine solution used for this study was $2 \mathrm{wt} \%$, and it was prepared using pure sodium chloride $(\mathrm{NaCl})$ provided by the Merck Company with properties listed in Table 1. 
Table 1 Properties of sodium chloride

\begin{tabular}{lllll}
\hline Description & Formula & $\begin{array}{l}\text { Molar mass } \\
(\mathrm{g} / \mathrm{mol})\end{array}$ & $\begin{array}{l}\text { Melting point } \\
\left({ }^{\circ} \mathrm{C}\right)\end{array}$ & $\begin{array}{l}\text { Density }(\mathrm{g} / \\
\left.\mathrm{cm}^{3}\right)\end{array}$ \\
\hline $\begin{array}{c}\text { Sodium } \\
\text { chloride }\end{array}$ & $\mathrm{NaCl}$ & 58.44 & 801 & 2.17 \\
\hline
\end{tabular}

First, a stock solution of $10 \mathrm{wt} \%$ was prepared. $1000 \mathrm{ml}$ of $2 \mathrm{wt} \% \mathrm{NaCl}$ solution was then prepared by diluting $200 \mathrm{ml}$ of the $10 \mathrm{wt} \%$ of the stock solution by adding $800 \mathrm{ml}$ of distilled water (stirrer used for mixing correctly).

\section{Surfactant solution}

\section{Surfactant solution preparation}

Table 2 shows the properties of the three surfactants used for this study. The concentration of surfactant for all tests was $0.5 \mathrm{wt} \%$ and the salinity was $2 \mathrm{wt} \%$.

Figure 1 shows the general structure of the first two anionic surfactants (AOS 14-16 and ENORDET 0332) which have been commercialized and employed in real fields for enhanced oil recovery (Barnes 2010; Wibbertmann et al. 2011). MFOMAX, on the other hand, is a mixture of anionic and amphoteric surfactants. It is a proprietary surfactant provided by PETRONAS Research Sdn Bhd (PRSB) which its structure has not been disclosed.

These surfactants were selected for this study due to their acceptability in the oil industry. The anionic surfactants incur lower adsorption losses in sandstones because their surface charge usually is negative, and mixed surfactants are used when some specific properties are desirable.

A stock solution of $5 \mathrm{wt} \%$ was prepared for each of the three surfactant solutions by adding an appropriate volume of pure surfactant to the distilled water as listed in Table 3. The amount of surfactant addition required to achieve the target concentration depended upon the active concentration of each pure surfactant.

The critical micelle concentration (CMC), an important parameter of a surfactant, was determined for the all three surfactants using the Accumet XL600 conductivity meter (by Fisher Scientific). The CMCs of the three surfactants were determined to be AOS $=0.033 \mathrm{wt} \%$, ENOR$\mathrm{DET}=0.016 \mathrm{wt} \%$, and MFOMAX $=0.062 \mathrm{wt} \%$.

These results were utilized in selecting a safe level of surfactant solution concentration to have the surfactant concentration above the $\mathrm{CMC}$ value to minimize the detrimental effect of adsorption in reducing the available active percentage of surfactant affecting foamability and foam stability.

The $1000 \mathrm{ml}$ of each surfactant solution ( $0.5 \mathrm{wt} \%$ surfactant concentration into $2 \mathrm{wt} \%$ salinity) were prepared for the surfactant characterization, core flood, and bulk foam stability tests.

\section{Surfactant solution characterization}

The density of the solution was measured at room temperature using Anton Paar DMA $4500 \mathrm{M}\left(0-3 \mathrm{~g} / \mathrm{cm}^{3}\right.$ range, $0.00005 \mathrm{~g} / \mathrm{cm}^{3}$ accuracy). Extreme care was exercised to remove all air bubbles from the testing tubes and waiting long enough for the system to stabilize. The surface tensions between nitrogen and surfactant solutions were measured
Table 2 Properties of surfactants used in this study

\begin{tabular}{|c|c|c|c|}
\hline Name & AOS 14-16 & ENORDET 0332 & MFOMAX \\
\hline Description & Alpha olefin sulphonate & Internal olefin sulphonate & $\begin{array}{l}\text { Mixed anionic } \\
\text { and amphoteric } \\
\text { surfactant }\end{array}$ \\
\hline Type & Anionic & Anionic & Mixed \\
\hline Physical state & Liquid & Liquid & Liquid \\
\hline $\begin{array}{l}\text { Molecular weight } \\
\text { average, } \mathrm{g} / \mathrm{mol}\end{array}$ & 315 & 414.19 & Proprietary \\
\hline$\%$ Activity & 33 & 31.30 & 20 \\
\hline $\mathrm{pH}$ & 10 & 14 & $6-6.5$ \\
\hline
\end{tabular}

Fig. 1 General structure of Alpha Olefin Sulfonate (AOS) (left) and Internal Olefin Sulfonate (ENORDET) (right) surfactants (Barnes 2010; Wibbertmann et al. 2011)

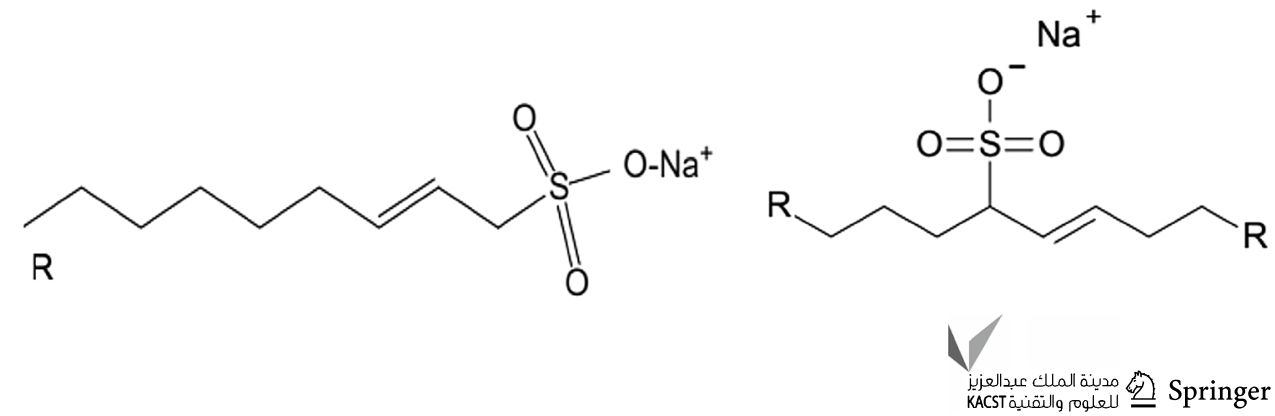


Table 3 Volumes used for making $1000 \mathrm{ml}$ of $5 \mathrm{wt} \%$ of surfactant stock solution

\begin{tabular}{llll}
\hline Name & AOS & ENORDET & MFOMAX \\
\hline Surfactant activity (\%) & $33 \%$ & $28.03 \%$ & $20 \%$ \\
Distilled water added (ml) & 848.5 & 821.6 & 750 \\
$\begin{array}{l}\text { Surfactant of given activity } \\
\quad 151.5\end{array}$ & 178.4 & 250 \\
added (ml) & & & \\
\hline
\end{tabular}

under atmospheric conditions by pendant drop method using IFT-700 Interfacial Tension Meter (by VINCI Technologies). Table 4 illustrates the results of densities and surface tension values. The density AOS and ENORDET are almost the same, whereas the density of MFOMAX is lower than the rest. Also, MFOMAX shows the highest reduction of surface tension among all.

\section{Bulk foam stability test}

The foam was generated using pure nitrogen $\left(\mathrm{N}_{2}\right)$ for this study. Though $\mathrm{CO}_{2}$ has a broader field application, it was avoided due to significant water solubility and corrosiveness issues, though it has been used in some research studies. The literature suggests that stronger foam can be generated with $\mathrm{N}_{2}$ which is also more compatible with different types of surfactants as compared to $\mathrm{CO}_{2}$ (Farajzadeh et al. 2009; Farzaneh and Sohrabi 2013). Figure 2 shows the graduated cylinders used in bulk foam stability tests with a steel tube inserted from the top to the base for gas injection from the bottom of the cylinder.

TECLIS Foam Scan (2018) (bulk foam analyzer) uses a fritted disk at the bottom through which gas is introduced into a partially surfactant-filled cylinder to generate foam. This study deliberately avoided using such a disk since the texture of the foam generated in this case, was controlled by the pore sizes, and the literature shows that texture influences stability in a way that finer foam has greater foam stability. The variations in the texture of foam may explain the reason why some studies in the past showed contradictory foam stability results.

The test was performed by pouring $50 \mathrm{ml}$ of the surfactant solution into a clean 1000-ml graduated cylinder of $6 \mathrm{~cm}$ diameter, and a steel tube was inserted to touch the bottom.
Fig. 2 The graduated cylinder used in bulk foam stability tests

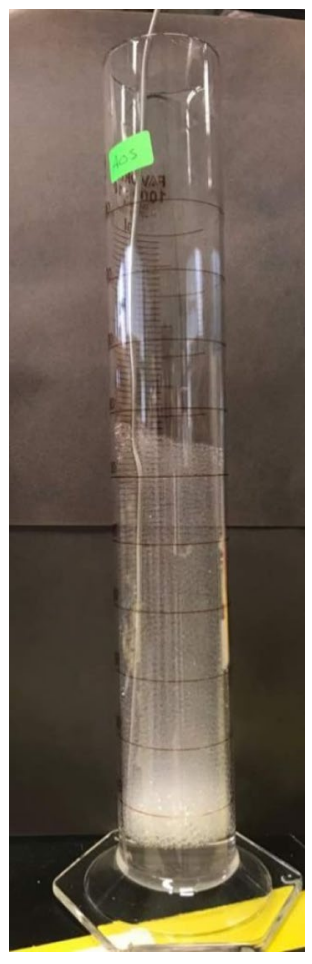

$\mathrm{N}_{2}$ gas from a cylinder was then injected at a rate of $30 \mathrm{~cm}^{3} /$ min (rate controlled by the gas regulator) through the steel tube. As the foam generation started, the height of the foam column started increasing. When the rise in foam height stops due to reaching equilibrium (new bubble generation equals bubble collapse), the $\mathrm{N}_{2}$ injection was stopped, the stopwatch was started to begin counting the lapse time, photographs were taken intermittently, and the height of the foam column was noted periodically. This process continued for about one day after which the test was closed.

\section{Coreflood foam stability tests}

Coreflood foam stability tests were performed in a commercial coreflooding system (BPS- 805 by Coretest Systems, Inc., CA, USA) using the core plug described in the next section. The system was equipped with a liquid pump, a gas mass controller for maintaining the desired gas injection rate, a differential pressure transducer, and a confining pressure system. The BPS-805 coreflooding system
Table 4 Surfactant solution characterization in term of its concentration and brine salinity

\begin{tabular}{lllll}
\hline Solution & $\begin{array}{l}\text { Surfactant concentra- } \\
\text { tion }(\mathrm{wt} \%)\end{array}$ & $\begin{array}{l}\text { Brine-NaCl salinity } \\
(\mathrm{wt} \%)\end{array}$ & Density $(\mathrm{g} / \mathrm{ml})$ & Tension $(\mathrm{mN} / \mathrm{m})$ \\
\hline Brine & 0 & 2 & 1.0121 & 72.68 \\
AOS & 0.5 & 2 & 1.0127 & 29.98 \\
ENORDET & 0.5 & 2 & 1.0120 & 29.07 \\
MFOMAX & 0.5 & 2 & 1.0090 & 28.25 \\
\hline
\end{tabular}



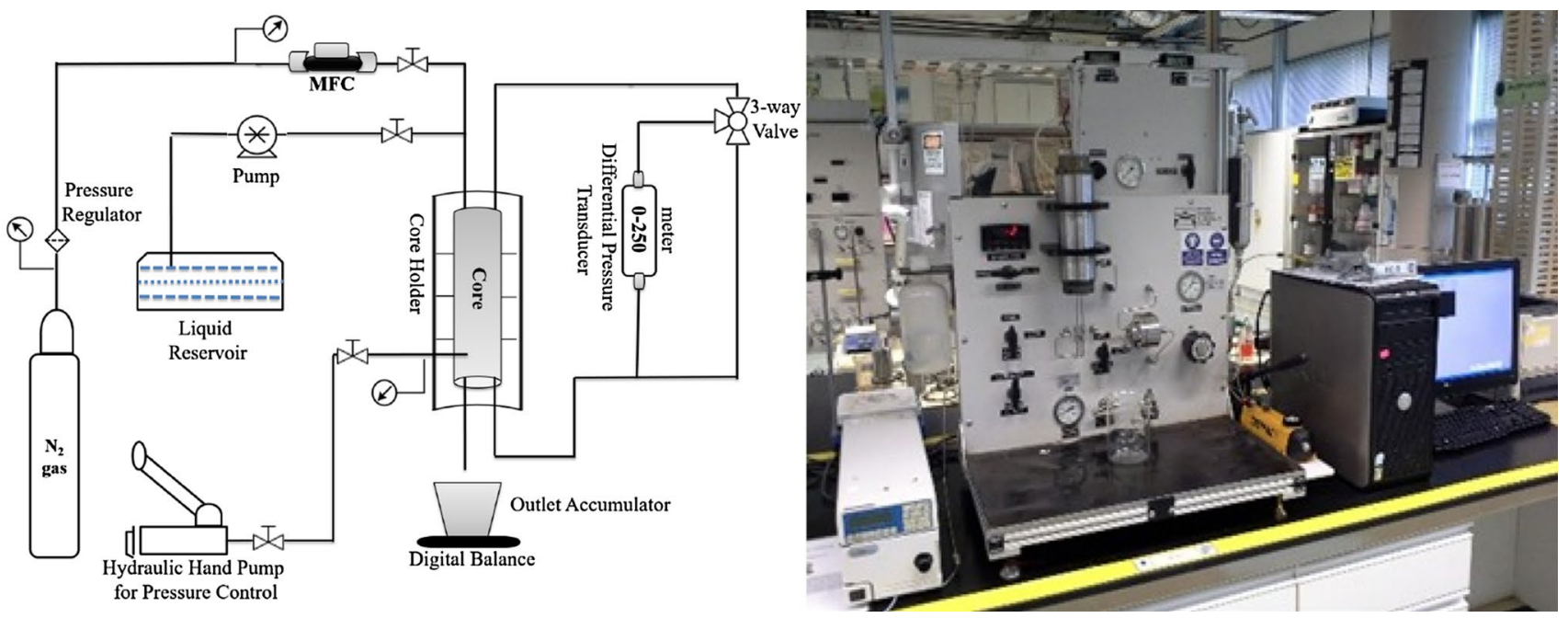

Fig. 3 Schematic and snapshot of BPS-805 coreflooding system

has been fully described by Kim and Lee (2017), except that the orientation of the core holder was vertical in this study. Figure 3 shows the schematic and the snapshot of the equipment.

The porosity and absolute permeability to gas were measured using the model M9170 poroperm system (by Grace Instrument), and the liquid permeability was determined using the BPS-805 coreflooding system with the singlephase liquid steady-state test. The residual water saturation $\left(S_{\mathrm{wr}}\right)$ was estimated using Timur's correlation for sandstones (Timur 1968) which correlates $S_{\mathrm{wr}}$ with absolute permeability and porosity, given in the following equation:

$S_{\mathrm{wr}}=3.5 \frac{\phi^{1.26}}{K^{0.35}}-1$,

where the porosity $(\phi)$ and permeability $(K)$ are in percent and $\mathrm{md}$, respectively.

In coreflood, the foaming performance was evaluated by drainage (unsteady state) experiments. The core was cleaned and saturated with a surfactant solution, and the test began by injecting $\mathrm{N}_{2}$ into the core. The differential pressure and cumulative gas injection volumes were noted vs. time. The apparent viscosity of foam was computed using the following equation (Ma et al. 2013):

$\mu_{\text {foam,app }}=\frac{K \nabla p}{U_{\mathrm{w}}+U_{\mathrm{g}}}$,

where $K$ is the permeability in Darcy, $\nabla p$ is the differential pressure gradient in $(\mathrm{atm} / \mathrm{cm})$, and $U_{\mathrm{w}}+U_{\mathrm{g}}$ is the total superficial velocity $(\mathrm{cm} / \mathrm{s})$.

\section{Core plug properties}

The core plug used for coreflood foam stability tests was commercially available (Kocurek Industries INC., TX, USA). The core used in this study was "Idaho Gray" sandstone cylindrical outcrop plug of $7.2 \mathrm{~cm}$ length and $3.7 \mathrm{~cm}$ in diameter. Table 5 lists the physical properties of the core plugs. The high permeability and porosity cores were chosen because of their suitability for foam studies as they are better for foam generation (Mannhardt et al. 2000) and they minimize the capillary end effects which could significantly affect the coreflooding results.

\section{Results and discussion}

\section{Bulk foam stability tests}

The bulk foam stability tests were performed on the three selected surfactants (same surfactants also used for coreflood foam stability tests) using the methods described in the previous section. The results are presented in Fig. 4 and show that the MFOMAX had the highest peak foamability since it was able to generate to a higher maximum foam volume of $990 \mathrm{ml}$ with $f_{\mathrm{g}}$ of $95 \%$, the medium performer was AOS with $640 \mathrm{ml}$ with $f_{\mathrm{g}}$ of $92 \%$, and the lowest performer was ENORDET with only $170 \mathrm{ml}$ with $f_{\mathrm{g}}$ of $71 \%$.

Residual foamability behavior was similar to the peak foamability, with MFOMAX having the highest residual foamability of $850 \mathrm{ml}$, AOS was the medium performer with $590 \mathrm{ml}$, and ENORDET was the lowest performer with only $50 \mathrm{ml}$. With regards to foam longevity, MFOMAX had the highest longevity and did not degrade before $30 \mathrm{~min}$. AOS showed medium longevity and started to degrade only after

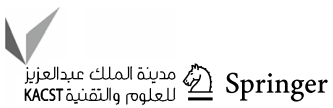


Table 5 Idaho gray sandstone core plug properties

\begin{tabular}{llllll}
\hline $\begin{array}{l}\text { Sam- } \\
\text { ple dry } \\
\text { weight } \\
(\mathrm{g})\end{array}$ & $\begin{array}{l}\text { Pore } \\
\text { volume } \\
(\mathrm{cc})\end{array}$ & $\begin{array}{l}\text { Porosity } \\
(\%)\end{array}$ & $\begin{array}{l}\text { Absolute } \\
\text { gas per- } \\
\text { meability } \\
(\mathrm{md})\end{array}$ & $\begin{array}{l}\text { Absolute } \\
\text { liquid } \\
\text { perme- } \\
\text { ability } \\
(\mathrm{md})\end{array}$ & $\begin{array}{l}\text { Residual } \\
\text { water } \\
\text { satura- } \\
\text { tion using } \\
\text { Timur's } \\
\text { correla- } \\
\text { tion \% }\end{array}$ \\
\hline 138.93 & 23.30 & 29.70 & 2974 & 903 & 14.27 \\
\hline
\end{tabular}

20 min while ENORDET showed the lowest longevity and started to degrade just after 2 min. Regarding foam decay rate, MFOMAX and ENORDET performed almost similar and took the longest time to drop from peak to valley.

AOS was the worst performer. After 20 min of longevity, it suddenly (in $6 \mathrm{~min}$ ) but only slightly dropped in volume by about $50 \mathrm{ml}$, after which it stayed stable until the end of the test. ENORDET had the slowest foam decay rate. After 2 min of longevity, foam began to decay non-linearly with time and dropped by $105 \mathrm{ml}$ in $24 \mathrm{~h}$. MFOMAX had almost similar foam decay rate as ENORDET and started to decay after $30 \mathrm{~min}$ and its volume reduced by $140 \mathrm{ml}$ in $23.5 \mathrm{~h}$. The above observations from bulk foam stability tests are summarized in Table 6 and shown in the clustered chart in Fig. 5.

A review of the peak foamability, residual foamability, and foam longevity attributes shows that the MFOMAX was the winner, the AOS was the runner-up, and the ENORDET was the least favorable. MFOMAX was also among the winners in having comparability low decay rate with ENORDET showing slightly better performance, whereas the AOS had drastically faster decay rate. Nonetheless, after a sudden drop in foam height, AOS maintained it residual foamability till the end. Thus, it can still be ranked as second best performing surfactant in these tests.

\section{Coreflood foam stability tests}

Three corefloods were performed using three different surfactants to rank them based on their foam stability. The characteristics of these surfactants were described in the previous section. The corefloods were performed using the single-slug SAG technique (the unsteady state surfactant drainage by gas injection), wherein the core was cleaned, dried, evacuated, and fully saturated with brine; which was then displaced by one of the three surfactant solutions. The tests were started by injecting gas and noting the pressure differential. All three surfactants were tested using the same core and under the same operating conditions as shown in Table 7 . The coreflood foam stability results are presented in Fig. 6.

For each test, differential pressure gradient was recorded (from which the apparent foam viscosity was calculated) with respect to the gas volume injected into the surfactantsaturated porous media. The pseudo apparent viscosity of foam $\left(\mu_{\text {foam }} / K_{\mathrm{rg}}\right)$ was derived in corefloods using Darcy's law. Since relative permeability curves for foam are not established, and the $S_{\mathrm{g}}$ varies during the test, $K_{\mathrm{rg}}$ could not have been determined. Under peak foaming conditions, however, $f_{\mathrm{g}}$ is only slightly less than 1 , and with that high gas saturation, $K_{\mathrm{rg}}$ is also close to 1 . Therefore, the pseudo apparent viscosity is expected to be very close or slighter higher than the apparent viscosity.

The early test data pertained to foamability behavior, i.e., pore volume injected before foaming started (delay in
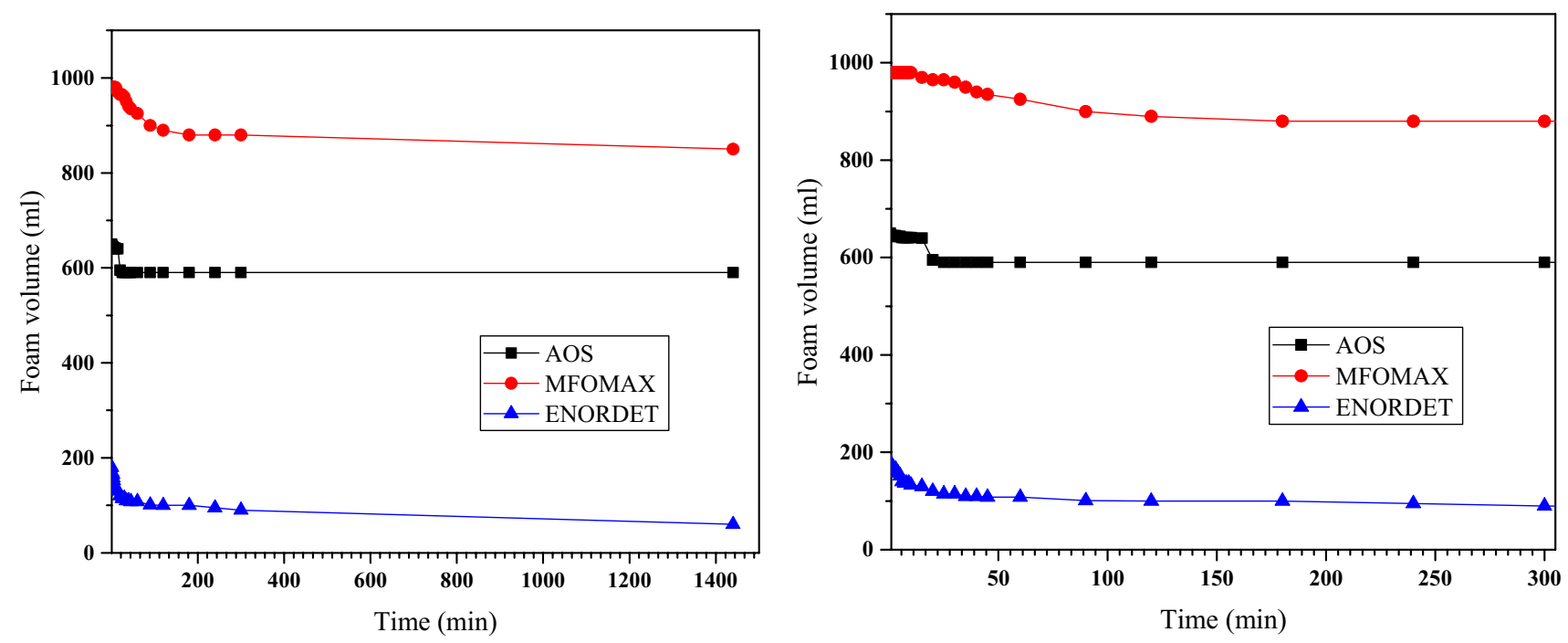

Fig. 4 The bulk foam stability tests for foam stability of the three selected surfactants, (left: complete test data, right: first 300 min of test data) 
Table 6 Summary of results from bulk foam stability tests

\begin{tabular}{|c|c|c|c|c|}
\hline Foam stability attribute & Observed parameter & MFOMAX & AOS & ENORDET \\
\hline Peak foaming & Foam volume (ml) & 990 & 640 & 170 \\
\hline Foam longevity & The time before decay started (min) & 30 & 20 & 2 \\
\hline Residual foaming & Foam volume after $24 \mathrm{~h}(\mathrm{ml})$ & 850 & 590 & 50 \\
\hline Foam decay rate & $\begin{array}{l}\text { The time required to drop foam vol- } \\
\text { ume from peak to valley (hours) }\end{array}$ & 23.5 & 0.1 & 24 \\
\hline Foam quality (\%) & $f_{\mathrm{g}}$ at the beginning & 95 & 92 & 71 \\
\hline Decay behavior & Qualitative observation & $\begin{array}{l}\text { Early transient: non-linear polyno- } \\
\text { mial; late transient: power law }\end{array}$ & Linear & $\begin{array}{l}\text { Early transient: non-linear } \\
\text { polynomial; late transient: } \\
\text { linear }\end{array}$ \\
\hline Decay behavior* & Quantitative observation & $a^{*}$ & $b^{*}$ & $c^{*}$ \\
\hline
\end{tabular}

Decay behavior of bulk foam stability test was divided into two sections as early and late transient for a better understanding of trends in Figs. 8 , 9 and 10.

$a *(\text { Early transient): Foam volume }=2887.36 \text { (Normalized time })^{2}-1590.83$ (Normalized time) +986.783

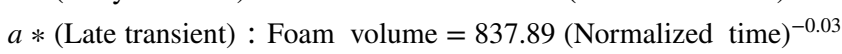

$b *($ Early transient $):$ Foam volume $=-2874.015$ (Normalized time) +641.43

$b *$ (Late transient) $:$ Foam volume $=-6.609$ (Normalized time $)+590$

$c *($ Early transient $):$ Foam volume $=37498.45$ (Normalized time $)^{2}-3239.45($ Normalized time $)+154$

$c *$ (Late transient): Foam volume $=-43.0480$ (Normalized time $)+92.6318$.

Fig. 5 The clustered column charts are displaying the results of the "bulk foam stability test" for the three surfactants. The chart on the left is for foam strength attributes, whereas the chart on the right is for the foam stability attributes
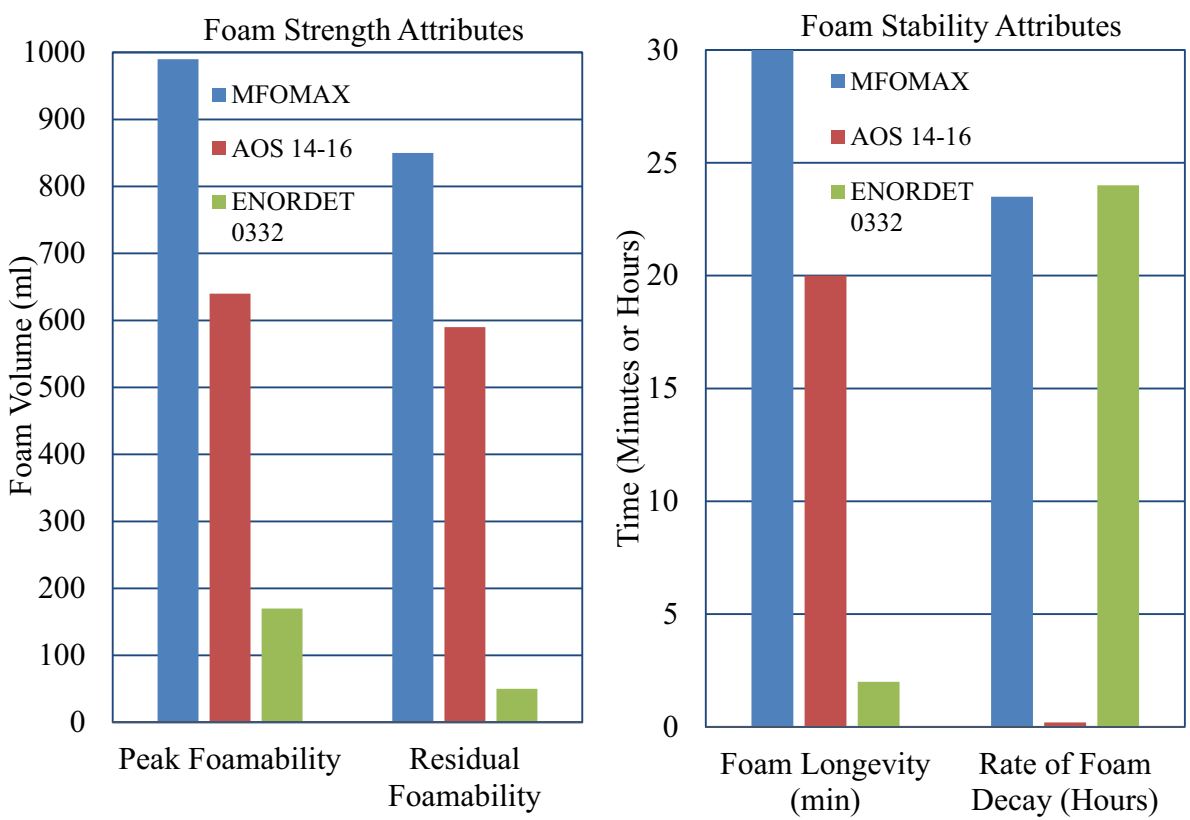

foaming) and between the start of foaming to reaching peak pressure drop (time to peak foaming). These data are not shown in Fig. 6 since those attributes cannot be determined in bulk foam stability tests. Bulk tests can only be used for testing foam stability and decay after the generation and peak foaming conditions. Therefore, to make a meaningful comparison, the pore volume injection was considered to be starting after foaming almost stabilized, i.e., zero PV injected on the figure indicates the point at which foam generation had virtually stabilized.

The coreflood foam stability tests showed that MFOMAX had the highest peak foamability since it was able to generate a pressure drop (DP) of up to 0.6 psig while AOS and ENORDET could only create pressure drop up to 0.36 and $0.35 \mathrm{psig}$, respectively. Overall, the results showed that MFOMAX is the best in foamability attributes, followed by AOS and after that ENORDET at the last. Looking at how 
Table 7 Operating conditions of corefloods

\begin{tabular}{ll}
\hline Parameters & Value \\
\hline Confining pressure (psi) & 1000 \\
Back pressure (psi) & 14.7 \\
Injection rate (cc/min) & 2 \\
Surfactant solution salinity & $2 \mathrm{wt} \%$ \\
Surfactant concentration & $0.5 \mathrm{wt} \%$ \\
Porosity (\%) & $29.7 \%$ \\
Permeability by gas (md) & 2974 \\
Permeability by liquid (md) & 903 \\
\hline
\end{tabular}

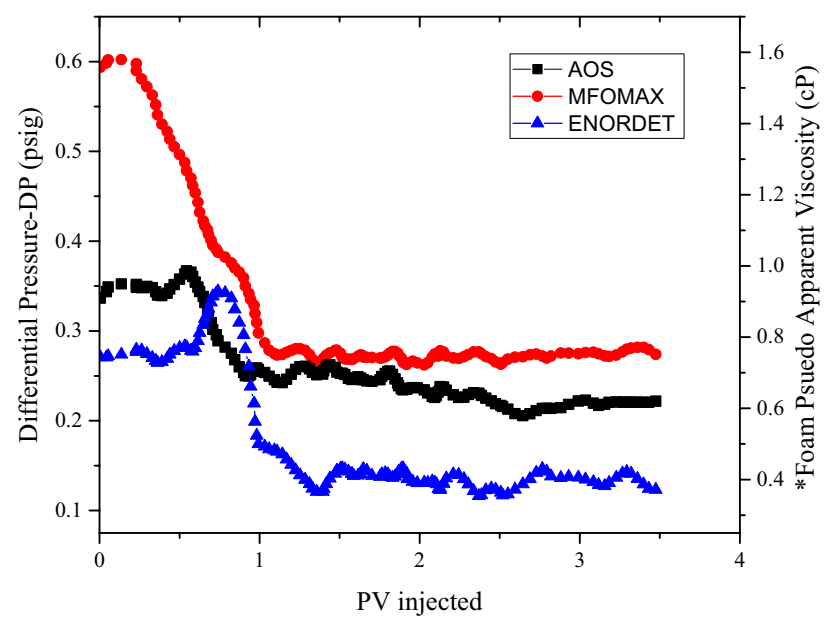

Fig. 6 The history of differential pressure change with the volume of gas injected into the core initially saturated with surfactant solution. The * indicates $\left(\mu_{\mathrm{foam}} / K_{\mathrm{rg}}\right)$ which is expected to be very close or slighter higher than the apparent viscosity

long the foam survived at peak foaming, ENORDET showed the highest foam longevity and did not start to decay until $0.8 \mathrm{PV}$ of gas injection. AOS had the medium longevity and did not begin to decay before $0.55 \mathrm{PV}$, whereas MFOAMX could only last $0.2 \mathrm{PV}$ before decay.

Regarding foam decay rate, MFOMAX had the slowest decay rate and took $1 \mathrm{PV}$ of gas injection from peak foaming to drop to the residual foaming. AOS had the medium decay rate and took $0.5 \mathrm{PV}$ to drop to the residual foaming, whereas ENORDET had the highest decay rate and took only $0.2 \mathrm{PV}$ of gas injection to go from the peak to residual.

With regards to the lowest pressure differential (DP at the valley), MFOMAX was again had the best performance with a DP of 0.275 psig, the AOS was the second best with a DP of $0.22 \mathrm{psig}$, and the ENORDET was the lowest performer with DP of $0.125 \mathrm{psig}$. The above observations are summarized in Table 8 and plotted as a cluster chart in Fig. 7.

The MFOMAX was the winner, the AOS was the runnerup, and the ENORDET was the least favorable with regards to peak foamability, residual foamability, and rate of foam decay attributes. The ranking was reverse, however, for foam longevity attribute where ENORDET was the best performer, AOS was the runner-up, and MFOMAX was the worst. It could have been due to its peak foaming DP being $71 \%$ and $62 \%$, respectively, higher than its competitors. Even after $0.8 \mathrm{PV}$ injection after the highest longevity surfactant (ENORDET), MFOMAX had a higher DP than other surfactants. Therefore, it can be claimed that MFOMAX was overall had the best performance compare to AOS and ENORDET.

\section{Side-by-side performance comparison of bulk and coreflood foam stability tests}

In previous sections, the performance of the three surfactants was analyzed separately for each test type with the idea to rank them in overall performance as well as in each of the attributes individually. In this section, the behavior of each surfactant as observed by both tests is presented side-by-side in Figs. 8, 9 and 10.

The timescale had to be normalized to show them on the same plot. Hence, for bulk foam stability test, the time data were divided by maximum time (1440 $\mathrm{min})$ to get a timescale from zero to one. Similarly, coreflood pore volumes were divided by the maximum pore volume. The two different foaming indicators, pressure drop (DP) for coreflood and foam volume $(\mathrm{ml})$, however, were depicted on two separate $y$-axes.

Figure 8 shows the foaming behavior of MFOMAX by the two tests. Only the early transient (140 min) in bulk foam stability test matched well with coreflood though later, the behavior diverged. The foam decay trend reduced continuously in coreflood test but slowed down and stabilized in bulk foam test. Decay behavior in bulk foam test encountered a change in structure, from "wet-foam" in which bubbles are not interfering, and liquid drainage is faster to "dry-foam" of the highly stable tetrahedral (weblike) structure. Contrarily, the foam flow in porous media is a dynamic process, which has a different decay mechanism as the data suggest as well as the previous studies have suggested (Farajzadeh et al. 2010).

Figure 9 shows the foaming behavior of AOS by the two tests. Relative less foaming (lower DP and Foam volume) was observed in both tests as compared to MFOMAX. The early transient behavior of bulk foam stability test was very short and did not match with the coreflood. After the early transient, the foam volume change was minor in bulk foam stability tests. The coreflood, on the other hand, had somewhat similar behavior as for MFOMAX. The reasons for the discrepancy are probably the same as explained above. 
Table 8 Summary of coreflood observations

\begin{tabular}{|c|c|c|c|c|}
\hline Foam stability attribute & Observed parameter & MFOMAX & AOS & ENORDET \\
\hline Peak foaming & Peak DP (psig) & 0.6 & 0.36 & 0.35 \\
\hline Foam longevity & Vol. inj. before decay started (PV) & 0.2 & 0.55 & 0.8 \\
\hline Residual foaming & DP at the valley (psig) & 0.275 & 0.22 & 0.125 \\
\hline Foam decay rate & $\begin{array}{l}\text { Vol. inj. between the start of DP drop } \\
\text { from peak to valley (PV) }\end{array}$ & 1 & 0.5 & 0.2 \\
\hline Foam quality $(\%)^{\mathrm{a}}$ (maximum) & $f_{\mathrm{g}}$ at the peak foaming & 86 & 86 & 86 \\
\hline Decay behavior & Qualitative observation & Linear & Linear & Non-linear polynomial \\
\hline Decay behavior** & Quantitative observation & $a^{* *}$ & $b^{* *}$ & $c^{* *}$ \\
\hline
\end{tabular}

${ }^{\text {a }}$ Since it is an unsteady state test, the quality of foam keeps changing. The maximum foam quality shown above is inferred from the maximum gas saturation possible in this core based on Timur's correlation. It is likely that maximum $f_{\mathrm{g}}$ shown above pertains to the peak foaming

Following decay behavior is result of fitting curve from Figs. 8, 9 and 10.

$a * *: \mathrm{DP}=-0.3243$ (Normalized time) +0.5694

$b * *: \mathrm{DP}=-0.1626$ (Normalized time) +0.3804

$c * *: \mathrm{DP}=0.22225$ (Normalized time) $)^{2}-0.43854$ (Normalized time) +0.3612

Fig. 7 The clustered column charts are displaying the corefloods results for the three surfactants. The chart on the left is for foam strength attributes, whereas the chart on the right is for the foam stability attributes
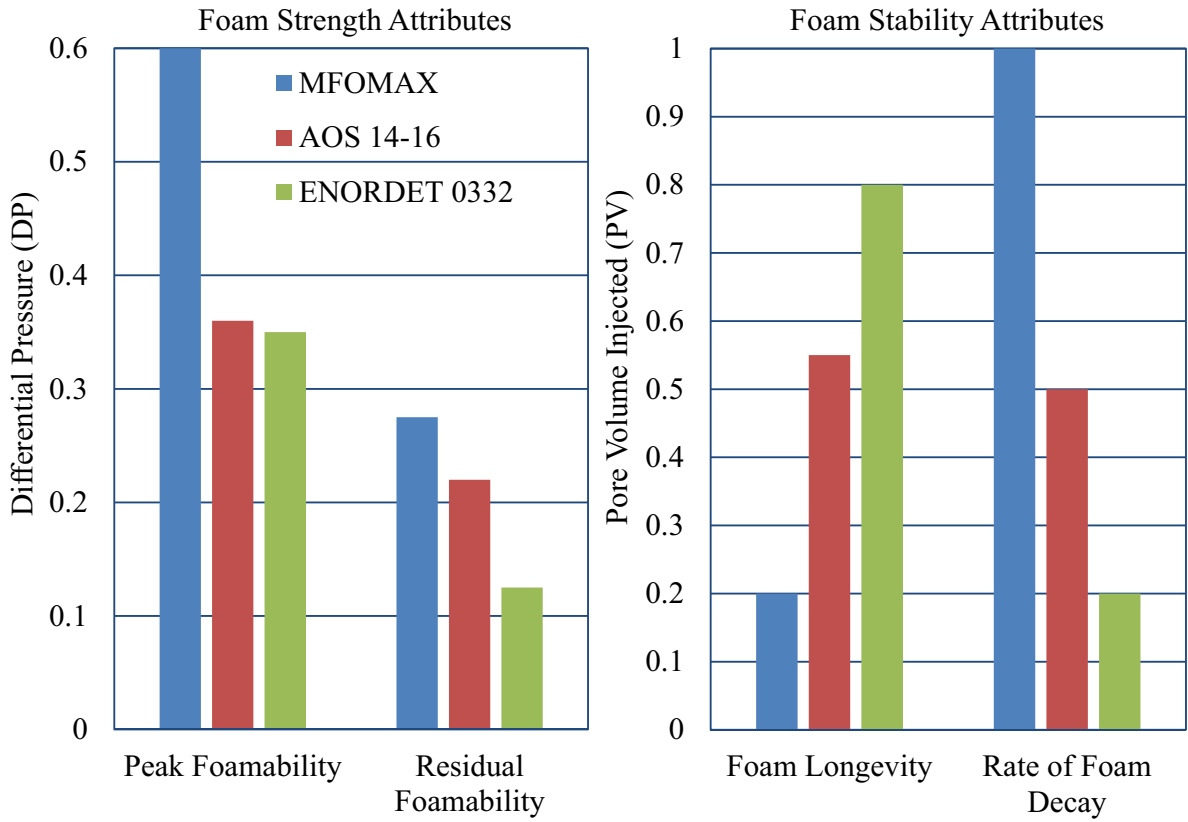

Figure 10 shows the foaming behavior of ENORDET by the two tests. The DP behavior in the coreflood test was somewhat similar and comparable to AOS coreflood test. The bulk foam stability test of ENORDET behaved differently than the previous two tests. In this case, the foam volume was significantly lower in the bulk foam stability test compare to AOS and MFOMAX. There was a sharp drop in foam volume during early transient before the decay rate significantly slowed down. This plot shows the shortcoming of using "half-life" as a screening criterion as the half-life, as shown in this chart, would have been very short and much before the "dry" foam was formed.

\section{Normalized performance comparison of bulk and coreflood foam stability tests}

The current industry practice for bulk foam stability tests is to use the half-life (the time taken for foam volume to reduce by half) is used as the primary factor distinguishing surfactant rankings. The difficulty with this practice is that bulk foam stability tests measure static stability, whereas the coreflood tests encounter dynamic stability. Because of this inherent difference, a new and more comprehensive approach will be used in comparing the two methods. In this approach, overall stability was deduced from four 


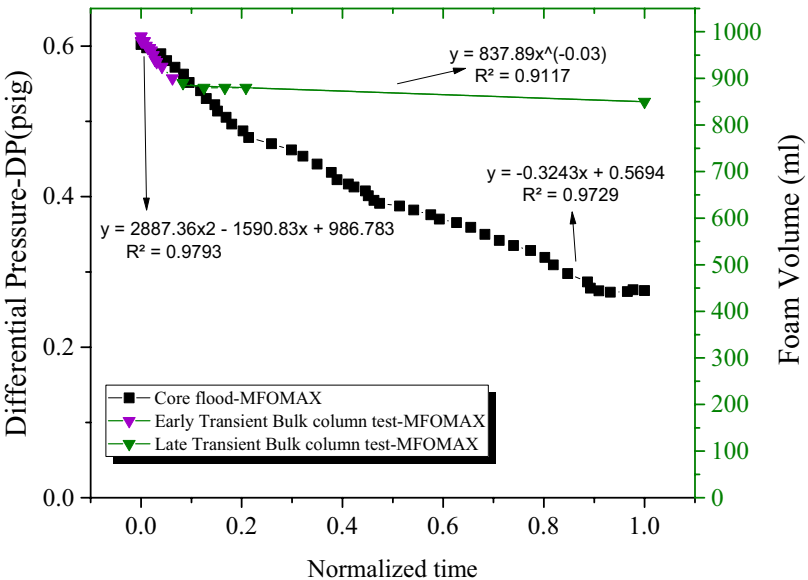

Fig. 8 Side-by-side performance comparison of bulk and coreflood foam stability tests of MFOMAX surfactant

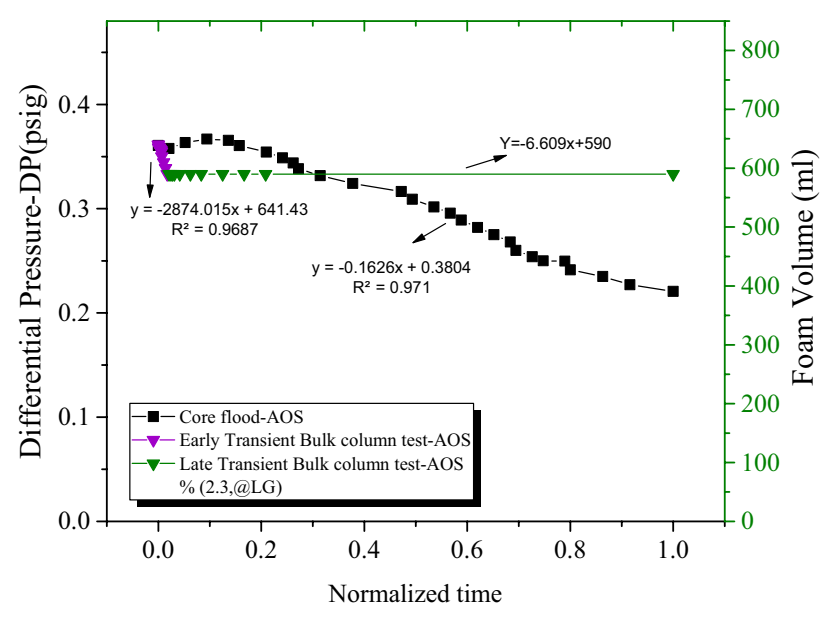

Fig. 9 Side-by-side performance comparison of bulk and coreflood foam stability tests of AOS surfactant

independent stability attributes: peak foamability, foam stability, the rate of foam decay, and residual foamability.

Above approach to ranking the surfactants were based on comparing the attributes performances of both tests individually. A side-by-side in-depth analysis of both tests together by comparing all the foaming attributes and their relative strengths among surfactant types is beneficial in screening the foaming surfactant. Since the quantitative measure of each attribute varies for each test type, such a comparison will require some intuitive techniques.

For comparing the attributes, it was necessary to normalize the data using the maximum value of each attribute from each test to yield a data range of $0-1$. Figure 11 shows and Table 9 compares the normalized foaming attributes between bulk and coreflood foam stability tests.

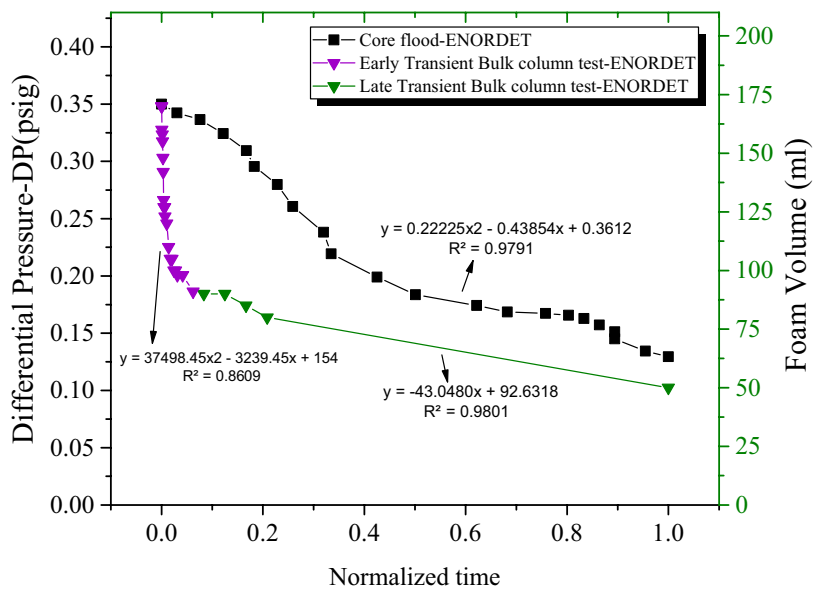

Fig. 10 Side-by-side performance comparison of bulk and coreflood foam stability tests of ENORDET surfactant

A careful side-by-side comparison of individual attributes on the juxtaposed charts (Fig. 11) reveals two shortcomings of the bulk foam stability test. First is that not all attributes are correctly ranked. Only the foamability (peak and residual) is correctly matched, whereas the foam stability attributes (longevity, decay rate) are not properly ranked. Second, even in the attributes that were correctly ranked, the relative merit is not correctly identified. AOS and ENORDET had almost similar peak foamability in corefloods but widely diverging in bulk foam stability tests. Such divergence is also evident in residual foamability.

Based on general observation from Fig. 11, ENORDET consistently showed differences between bulk and core tests, whereas MFOMAX and AOS were fairly consistent between bulk and core tests and it is due to its molecular structure that leads to a higher tendency to form micelles or aggregates in the liquid phase. This behavior results in a reduced number of surfactant molecules at the interface affecting their performance in maintaining the lamellae strength. AOS, on the other hand, is an anionic surfactant with straight carbon chain structure (no branching) leading to the better molecular arrangement at the interface. MFOMAX is a mixture of anionic and amphoteric surfactants having stronger steric interfacial forces between the surfactant molecules at the surface of lamellae which improves foam stability and strength.

Therefore, it can be inferred that the use of bulk foam stability tests should be restricted to screening out significantly lower performers. Screening out surfactants with closely matching performance by bulk foam stability tests alone is open to the risk of eliminating competent surfactants. 
Fig. 11 The clustered column charts are comparing bulk foam test with coreflood results for all four foaming attributes: peak foamability, foam longevity, foam decay rate, and residual foamability. The data have been normalized from 0 to 1 using the maximum value of each attribute

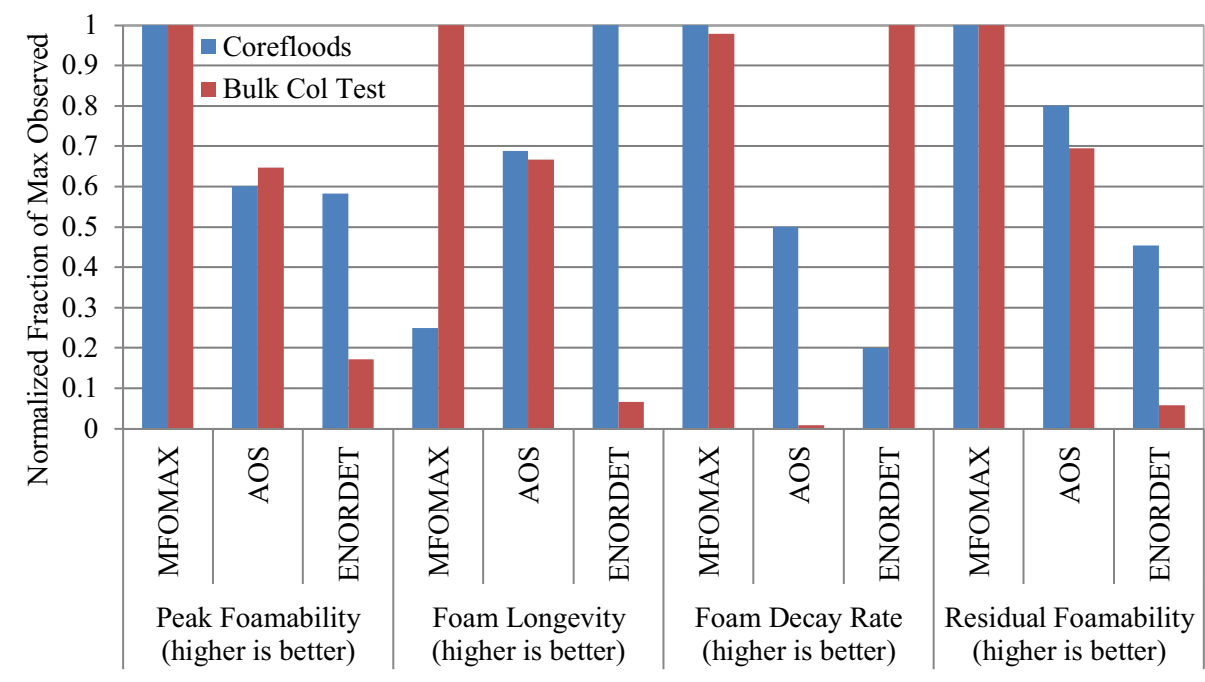

Table 9 Comparison of normalized foaming attributes between corefloods and bulk foam stability tests

\begin{tabular}{|c|c|c|c|c|c|c|c|c|}
\hline \multirow[t]{2}{*}{ Attribute } & \multicolumn{4}{|l|}{ Coreflood foam stability tests } & \multicolumn{4}{|l|}{ Bulk foam stability tests } \\
\hline & Observed parameter & MFOMAX & AOS & ENORDET & Observed parameter & MFOMAX & AOS & ENORDET \\
\hline Peak foamability & Peak DP (psig) & 1 & 0.6 & 0.58 & Peak foam volume (ml) & 1 & 0.65 & 0.17 \\
\hline Foam longevity & $\begin{array}{l}\text { Onset of foam collapse } \\
(\mathrm{PV})\end{array}$ & 0.25 & 0.68 & 1 & Stable for (min) & 1 & 0.66 & 0.067 \\
\hline Foam decay rate & $\begin{array}{l}\mathrm{PV} \text { required to drop DP } \\
\text { from peak to valley }(\mathrm{PV})\end{array}$ & 1 & 0.5 & 0.2 & $\begin{array}{l}\text { Time required to drop } \\
\text { foam volume from peak } \\
\text { to valley (min) }\end{array}$ & 0.98 & 0.01 & 1 \\
\hline Residual foamability & DP at the valley (psig) & 1 & 0.8 & 0.45 & Volume after 24 h (ml) & 1 & 0.7 & 0.06 \\
\hline
\end{tabular}

\section{Conclusion}

After an in-depth analysis of foaming behavior of three surfactants (MFOMAX, AOS, and ENORDET) as observed by two different foam screening methods (bulk and coreflood foam stability tests), the following can be concluded:

1. The new rigorous technique successfully analyzed the bulk foam stability performance of surfactants at ambient, oil-free conditions.

2. Bulk foam stability tests identified the highest and lowest performing surfactants correctly, though the individual attributes (peak foamability, foam longevity, foam decay rate, and residual foaming) did not always correlate well with coreflood tests. However, it can still be used as a preliminary screening tool to reduce the number of surfactants for further testing by a more reliable technique (coreflood).

3. The delay in onset of foaming and time required to reach peak foaming were two of the attributes that could be determined in coreflood tests but not in bulk foam stability tests.
4. The foam decay behaviors of coreflood and bulk foam stability tests were quite different. Foam decayed somewhat linearly in corefloods but had two distinct regimes in bulk foam tests. There was a rapid decay during early transient thought to be "wet foam" and a much slower decay during the late transient of "dry foam." Without knowing the regime, using half-life as a measure of foam quality is prone to errors.

Acknowledgements The authors gratefully acknowledge the financial support from the Shell-TU Delft-UTP collaborative project under Grant no: 0153AB-DA2. The research was conducted at the COREOR center of University Teknologi PETRONAS.

Open Access This article is distributed under the terms of the Creative Commons Attribution 4.0 International License (http://creativeco mmons.org/licenses/by/4.0/), which permits unrestricted use, distribution, and reproduction in any medium, provided you give appropriate credit to the original author(s) and the source, provide a link to the Creative Commons license, and indicate if changes were made. 


\section{References}

Andrianov A, Farajzadeh R, Mahmoodi Nick M, Talanana M, Zitha PLJ (2012) Immiscible foam for enhancing oil recovery: bulk and porous media experiments. Ind Eng Chem Res 51(5):2214-2226

Barnes JR, Dirkzwager H, Smit JR, Smit JP, On A, Reinaldo Navarrete C, Ellison BH, Buijse, MA (2010) Application of internal olefin sulfonates and other surfactants to eor. part 1: structureperformance relationships for selection at different reservoir conditions. Society of Petroleum Engineers, pp 1-16. https://doi. org/10.2118/129766-MS

Bergeron V, Fagan ME, Radke CJ (1993) Generalized entering coefficients: a criterion for foam stability against oil in porous media. Langmuir 9(7):1704-1713

Bond DC, Holhrook OC, Lake C (1958) States 1 2,866,507, pp 2-4

Farajzadeh R, Andrianov A, Bruining H, Zitha PLJ (2009) Comparative study of $\mathrm{CO}_{2}$ and $\mathrm{N}_{2}$ foams in porous media at low and high pressure-temperatures. Ind Eng Chem Res 48(9):4542-4552

Farajzadeh R, Andrianov A, Zitha PLJ (2010) Investigation of immiscible and miscible foam for enhancing oil recovery. Ind Eng Chem Res 49(4):1910-1919

Farzaneh SA, Sohrabi M (2013) SPE 164917 a review of the status of the foam applications in enhanced oil recovery. SPE J. https://doi. org/10.2118/164917-MS

Franklin J, Orr M (2007) Theory of gas injection processes. Tie-Line, Holte

Green DW, Willhite GP (1998) Enhanced oil recovery. Henry L. Doherty Memorial Fund of AIME, Society of Petroleum Engineers, Richadson

Hanssena JE, Kristiansen TS (1994) Oil interaction with foams under static and flowing conditions in porous media. Colloids Surf A $82: 129-140$

Hirasaki GJ (1985) Mechanisms of foam flow in porous media: apparent viscosity in smooth capillaries. Soc Pet Eng J 25(2):176-190

Jones SA (2018) Foam flow in a model porous medium: I. the effect of foam coarsening. Soft Matter. https://doi.org/10.1039/C7SM0 $1903 \mathrm{C}$

Jones SA, van der Bent V, Farajzadeh R, Rossen WR, Vincent-Bonnieu $S$ (2015) Small core flood experiments for foam EOR-screening surfactant applications. In: 18th European symposium on improved oil recovery. https://doi.org/10.3997/2214-4609.20141 2126

Jones SA, van der Bent V, Farajzadeh R, Rossen WR, Vincent-Bonnieu S (2016) Surfactant screening for foam EOR: correlation between bulk and core-flood experiments. Colloids Surf A Physicochem Eng Asp 500:166-176

Kim C, Lee J (2017) Experimental study on the variation of relative permeability due to clay minerals in low salinity water-flooding. J Pet Sci Eng 151:292-304. https://doi.org/10.1016/j.petro 1.2017.01.014

Lake LW (1996) Enhanced oil recovery, 1st edn. Prentice Hall, Upper Saddle River

Li J, Pan R, Guo B, Shan J (2014) Thermal stability of brine foams for shale gas drilling. J Nat Gas Sci Eng 17:131-135

Ma K, Lopez-Salinas JL, Puerto MC, Miller CA, Biswal SL, Hirasaki GJ (2013) Estimation of parameters for the simulation of foam flow through porous media. Part 1: the dry-out effect. Energy Fuels 27(5):2363-2375

Mannhardt K, Novosad J, Schramm L (2000) Comparative evaluation of foam stability to oil. SPE Reserv Eval Eng 3(1):19-22

Namani M, Kleppe J, Høier L, Karimaie H, Torsæter O (2012) Analytical model for zones distributions in non-horizontal miscible WAG injection. Energy Environ Res 2(2):159-167

Osei-bonsu K, Grassia P, Shokri N (2017) Relationship between bulk foam stability, surfactant formulation and oil displacement efficiency in porous media. Fuel 203:403-410. https://doi. org/10.1016/j.fuel

TECLIS SCIENTIFIC (2018) France, Feb. 08. https://www.teclis-scien tific.com/foam-analysis.

Timur A (1968) An investigation of permeability, porosity, and residual water saturation relationships for sandstone reservoirs, vol 9. Society of Petrophysicists and Well-Log Analysts

Van Der Bent VJ (2014) Comparative study of foam stability in bulk and porous media. AES/PE:14-24

Vikingstad AK, Aarra MG (2009) Comparing the static and dynamic foam properties of a fluorinated and an alpha olefin sulfonate surfactant. J Pet Sci Eng 65(1-2):105-111

Wibbertmann A, Mangelsdorf I, Gamon K, Sedlak R (2011) Toxicological properties and risk assessment of the anionic surfactants category: alkyl sulfates, primary alkane sulfonates, and $\alpha$-olefin sulfonates. Ecotoxicol Environ Saf 74(5):1089-1106

Zhang ZF, Freedman VL, Zhong L (2009) Foam transport in porous media-a review. US Dept of Energy Report no. PNNL-18918, The United States. https://doi.org/10.2172/1016458

Publisher's Note Springer Nature remains neutral with regard to jurisdictional claims in published maps and institutional affiliations.

\section{Affiliations}

\section{Negar Hadian Nasr ${ }^{1}$ (D) Syed M. Mahmood ${ }^{1} \cdot$ Hamed Hematpur $^{2}$}

Negar Hadian Nasr

negar.hadian@gmail.com

1 Department of Petroleum Engineering, Universiti Teknologi PETRONAS, Seri Iskandar, Malaysia
2 Petroleum Department, Research Institute of Petroleum Industry, Tehran, Iran 